\title{
EIN AUTOMATISCHER APPARAT ZUR BESTIMMUNG DER AUSTAUSCHBAREN IONEN, INSBESONDERE PHOSPHAT- IONEN, IM BODEN MITTELS IONENAUSTAUSCHER
}

\author{
Eugen BARKOFF
}

Landwirtschaftliche Versuchsanstalt, Abteilung für Agrikulturchemie und-physik, Tikkurila, Finnland

Eingegangen am 7. 7. 1956

Die Menge des austauschbaren Phosphates, besonders in sauren Böden, ist eines der wichtigsten Kriterien für den Phosphorzustand des Bodens.

Wie bekannt, gibt es verschiedene Methoden das austauschbare Phosphat zu bestimmen. Meistens hat man zum Extrahieren desselben entweder alkalische Lösungen (z.B. $\mathrm{NaOH}$ und $\mathrm{K}_{2} \mathrm{CO}_{3}$ ) oder neutrale Ammoniumfluoridlösungen benutzt. SHEARD \& CALDWEll (3) haben das austauschbare Phosphat im Boden durch Schütteln einer fein zermahlenen Bodenprobe im Wasser mit einer etwas gröberen Fraktion eines Anionenaustauschers zu bestimmen versucht. Nach der Schütteln wurde das Harz durch Spülung mit destilliertem Wasser auf einem Sieb von der Bodensubstanz getrennt, wobei diese durch das Sieb passiert wird und das Harz darauf liegenbleibt. Das Phosphat wurde dann aus dem Harz eluiert und kolorimetrisch bestimmt.

Da das Harz auf diese Weise von der Bodensubstanz ohne Phosphorverluste quantitativ schwer zu trennen ist und da diese Methode sonst einige andere Nachteile einschliesst, suchte ich nach einem automatischen Apparat, bei dem die Lösung durch das ganze System kontinuierlich passieren sowie Harz und Bodenprobe voneinander getrennt sich vorfinden würden.

Eine einfache kontinuierliche Versickerungsvorrichtung hat LEEs (2) gebaut. Die Zirkulation der Lösung ist dabei so angeordnet, dass die Lösung mit Luftblasen, mit Hilfe des Einsaugens durch ein Glasröhrchen, in eine gewisse Höhe aus einem Gefäss gehoben wird. Von dort fliesst diese Lösung durch die in einem Glasrohr befindliche Bodenprobe in dasselbe Gefäss zurück. Die ganze Vorrichtung 


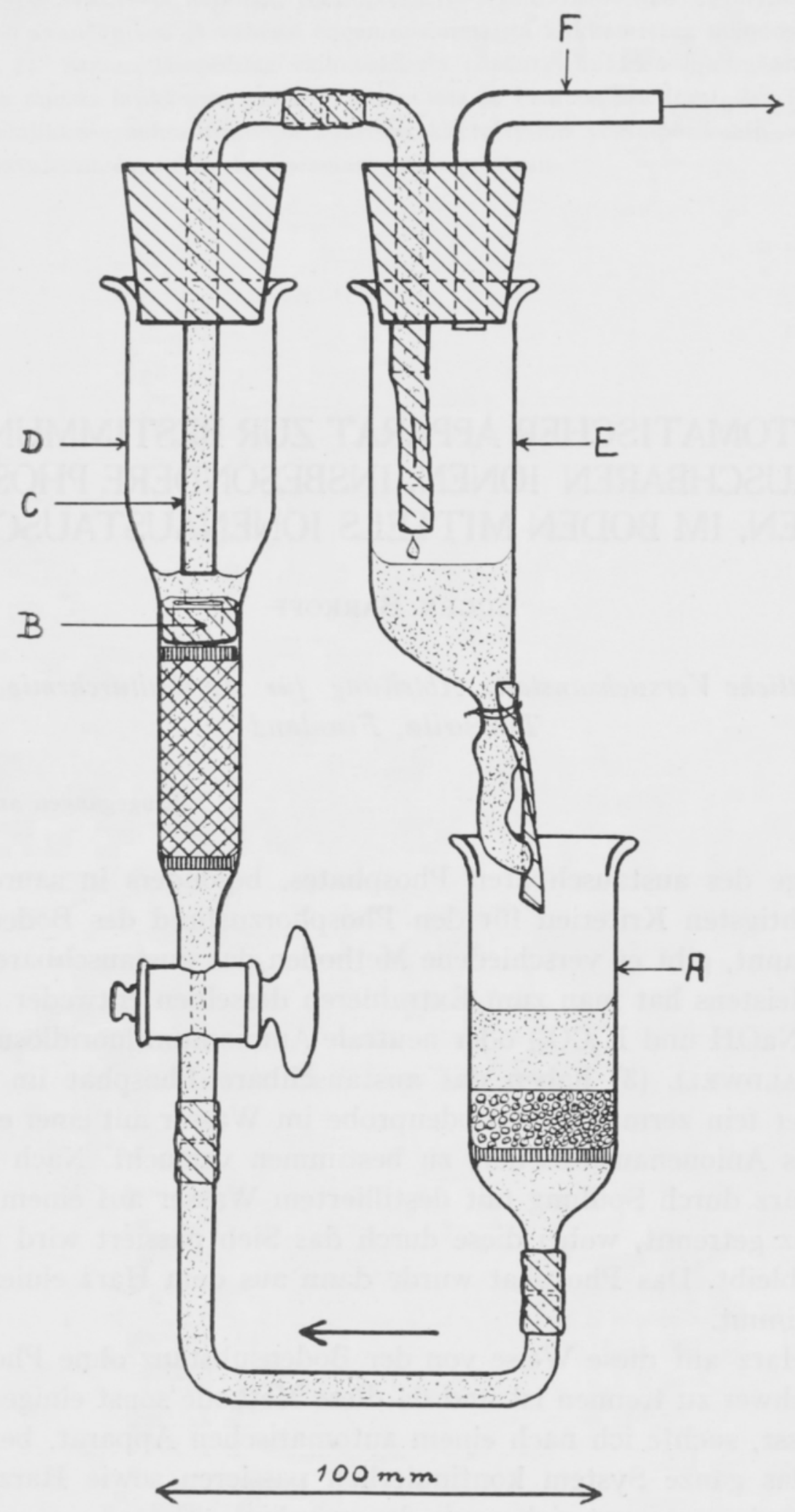

Abb. 1. Apparat während der Vakuumphase.

$\mathrm{A}=$ Trichter mit eingeschmolzener Sinterplatte für Bodenprobe; $\mathrm{B}=$ Stumpf eines Vakuumgummi schlauches; $\mathrm{C}$ und $\mathrm{F}=$ Glasröhrchen; $\mathrm{D}=$ Glasrohr mit Harz zwischen Sinterplatten; $\mathrm{E}=$ Trichter mit einem Gummischeibeventil. Innerhalb desselben hängt ein Bunsensches Ventil. 
befindet sich während des Betriebes unter Vakuum, und nur die Luftblasen dringen durch ein Kapillarrohr in dieses System ein.

Weil diese Vorrichtung für unseren Zweck nicht effektiv genug ist, habe ich den untenbeschriebenen automatischen Apparat konstruiert.

\section{Beschreibung des automatischen Apparates}

Die Konstruktion meines Apparates geht am besten aus den Abbildungen 1 und 2 hervor.

$A$ besteht aus einem etwa $50 \mathrm{ml}$ fassenden, zylinderförmigen Trichter, in den unten eine grobe Sinterplatte, G 1, eingelötet ist. Dieser Trichter ist durch Gummischläuche zuerst mit einem gebogenen Glasröhrchen und dann mit dem eigentlichen Ionenaustauscherrohr $D$ vereinigt.

$D$ ist ein etwa $200 \mathrm{~mm}$ langes Glasrohr. In der Mitte hat es einen Durchmesser von etwa $20 \mathrm{~mm}$. Nach oben ist es zu einem zylinderförmigen Trichter erweitert, nach unten verengert es sich zu einem schmalen Glasrohr und ist mit einem Glashahn versehen. Unten, in dem mittleren Teile des Glasrohres $D$ über dem Glashahn, befindet sich auch eine eingelötete grobe Sinterplatte G 1. Auf diese Platte wird lufttrockenes Harz gelegt. Darauf wird wieder eine gleiche, lose Sinterplatte, die gut in den mittleren Teil des Rohres $D$ hinein geht, gelegt. Um das Harz während des Betriebes des Apparates festzuhalten, wird das obere Ende des mittleren Teiles in dem Rohre $D$ z.B. mit einem passenden Stückchen eines Vakuumgummischlauches, $B$, zugeschlossen. Das ist nur dann möglich, wenn das Volumen des Harzes sich während des Vorgangs nicht verändert. Im anderen Falle muss man z.B. eine elastische Feder aus rostfreier Substanz zu diesem Zweck anwenden.

Das erweiterte obere Ende des Rohres $D$ ist mit einem Gummipropfen, durch den das gebogene Glasröhrchen $C$ führt, verchlossen. Das untere Ende des Glasröhrchens $C$ reicht beinahe bis an den Vakuumschlauch $B$ hinab, und sein oberes

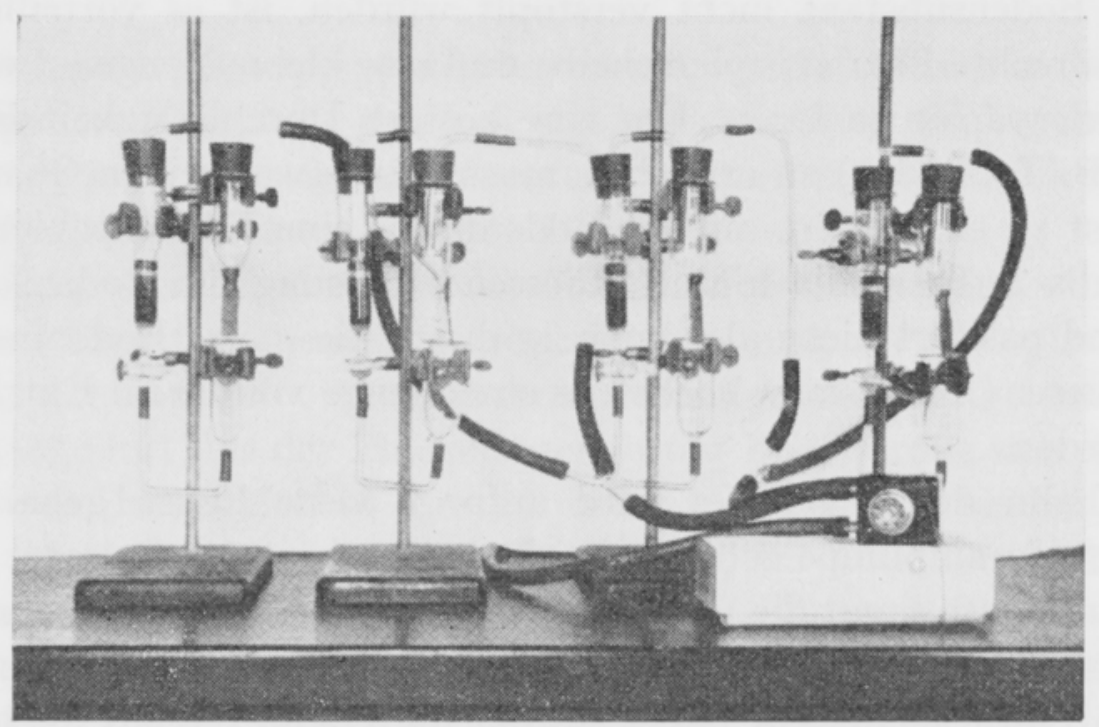

Abb. 2. Eine Reihe von 4 Extraktionsapparaten mit einem gemeinsamen Membranpulsator. 
Ende ist durch einen Gummischlauch mit dem anderen gebogenen Glasröhrchen und durch diesen mit dem Trichter $E$ verbunden.

$E$ ist ein zylinderförmiger Glastrichter, dessen unteres Röhrchen so gebogen ist, dass seine Öffnung sich in vertikaler Stellung befindet. Vor der Öffnung hängt eine dünne Gummischeibe. Um den Trichter $A$ leichter zugänglich zu bekommen, ist der Trichter $E$ asymmetrisch konstruiert. Dieser Trichter $E$ ist oben mit einem Gummipropfen, durch den zwei gebogene Glasröhrchen führen, verschlossen. An dem unteren Ende des einerı von diesen Röhrchen, das mit dem Röhrchen $C$ verbunden ist, ist ein Bunsensches Ventil angebracht. Das andere Glasröhrchen $F$ ist mittels eines Vakuumgummischlauches mit einem Membranpulsator einer AlfaLaval-Melkmaschine vereinigt, welcher seinerseits mit der Vakuumpumpe verbunden ist.

Der Pulsator ist ein Mechanismus, der eine regelmässige Abwechslung zwischen Vakuum und Luftdruck zustande bringt (JAnsson (1), S. 55-58). Die Geschwindigkeit dieser Abwechslungen im Pulsator kann nach belieben geregelt werden. Ein und derselbe Pulsator ist imstande, wenigstens vier solche Apparate gleichzeitig zu bedienen. In diesem Zusammenhang wäre noch zu erwähnen, dass der Puisator verhältnismässig billig ist.

Um den Kreislauf der Lösung im Apparat genauer regeln zu können, sind an beiden Seiten des Glashahnkegels, an dessen Oberfläche in Querrichtung, sich nach aussen von dem Loch in derselben Richtung verengernde Einschnitte gemacht. Auf diese Weise verursacht eine kleine Veränderung in der Stellung des Hahnkegels keine so grosse Veränderung bei der Zirkulationsgeschwindigkeit der Lösung wie bei Anwendung des normalen Hahnkegels.

\section{Beschreibung der Arbeitsmethodik bei dem automatischen Apparat}

Bei Anwendung des Apparates verfährt man auf folgende Weise: In den Trichter $A$ wird eine Bodenprobe und die Extraktionslösung getan. Damit die Poren des Sinters von der Bodensubstanz nicht verstopft würden, ist es vorteilhaft, auf dem Sinter eine passrechte Filtrierpapierscheibe und eine kleine Menge (etwa $200 \mathrm{mg}$ ) zermahlenes Filtrierpapier zu legen. Um eine bessere Durchlässigkeit bei feinen Bodenproben (z.B. Tonboden) zu erreichen, muss man etwas reinen Quarzsand hinzufügen. Auch ist zu empfehlen, auf die Bodenprobe eine Filtrierpapierscheibe zu legen. Andernfalls zerfrisst die hinunterfliessende Lösung die Bodenschicht nur an einer Stelle und passiert nicht gleichmässig durch die ganze Bodenmenge. Bei Apparaten von dieser Grösse kann höchstens eine Menge von $50 \mathrm{ml}$ Extraktionslösung gebraucht werden.

Der Glashahn des Apparates wird anfangs verschlossen gehalten, während der Hahn der Vakuumpumpe geöffnet ist. Dabei beginnt der Pulsator zu fungieren. Bei der Vakuumphase saugt die Gummischeibe des Trichters $E$ sich an die Öffnung des Röhrchens fest und macht sie zu. Dabei ist hingegen das Bunsensche Ventil offen. Der Unterdruck entsteht dabei sowohl im Trichter $E$ als auch im $\operatorname{Rohr} D$. Während der Luftdruckphase schliesst sich das Bunsensche Ventil und unterhält 
den Unterdruck im Rohre $D$, obgleich auch Luftdruck im Trichter $E$ entsteht. Auf diese Weise hält sich der Unterdruck fortdauernd im Rohre $D$.

Nun öffnet man den Glashahn so weit, dass die Lösung mit passender Geschwindigkeit zu zirkulieren beginnt. Bei der Vakuumphase steigt die Lösung von unten durch das Harz in das Rohr $D$ bis zu dem unteren Ende des Röhrchens $C$. Danach passiert die Lösung dieses Röhrchen und das Bussexsche Ventil und geht in den Trichter $E$ hinüber.

Die während der Vakuumphase in den Trichter $E$ angesammelte Lösung wird bei der Luftdruckphase in den Trichter $A$ mit eigenem Druck hinunterfliessen. Gleichzeitig steigt im Rohr $D$ die Lösung bis etwa zur Hälfte des breiten Teiles, dann geht sie während der folgenden Vakuumphase sofort in den Trichter $E$ hinüber. Als ein die Zirkulationsgeschwindigkeit der Lösung beschränkender Faktor wirkt der Umstand, dass sich in dem Trichter $E$ während der Vakuumphase niemals mehr Lösung sammeln darf als diejenige Menge, die während der folgenden Phase von dort wegfliesst. Sonst besteht Gefahr, dass die Lösung in die Saugvorrichtung hinüberfliesst. Als normale Zirkulationsgeschwindigkeit für diesen Apparat berechnet man ungefähr 7 Liter in der Stunde.

Anfangs werden die Ionen aus der Lösung an dem unteren Teil der Harzsäule festgelegt. Bei längerer Zirkulation wandern die Ionen allmählich nach oben, und bei Erreichen des Gleichgewichtes sind sie gleichmässig in der ganzen Harzmenge verteilt. Die Eluierung wird in der entgegengesetzten Richtung, von oben nach unten, ausgeführt.

Für die Eluierung wird das Rohr $D$ von dem Apparat entfernt, und um die Oberfläche der Lösung dabei nicht zu sehr sinken zu lassen, wird ein Siphon, dessen freies Ende sich über der Oberfläche der Harzsäule befindet, mit dem unteren Röhrchen des Rohres $D$ verbunden. Auf diese Weise kommt die Luft niemals unversehens in die Harzsäule, was die vollständige Eluierung des Harzes stören würde.

\section{Einige Grundsätze bei der Anwendung des Apparates}

Den oben beschriebenen Apparat haben wir vorläufig nur für die Bestimmung des austauschbaren Phosphats im Boden gebraucht. Ohne die Ergebnisse der noch fortgehenden Untersuchung im einzelnen zu beschreiben, werde ich hier nur einige Grundsätze analytisch-technischer Art berühren.

Prinzipiell ist diese Methode in der Tat dieselbe wie bei Sheard \& Caldwell (3): In beiden Fällen tauschen die Anionen der Lösung die Phosphationen der Bodenprobe aus, die ihrerseits an den Anionenaustauscher festgelegt werden. Das setzt sich so lange fort, bis der Phosphatgehalt der Bodenprobe sich so sehr vermindert und der des Harzes sich so sehr vermehrt hat, dass die beiden festen Phasen sich mit der Phosphatkonzentration der Lösung im Gleichgewicht befinden. In beiden Fällen bleibt in der Bodensubstanz noch eine mehr oder weniger grosse Menge des gleichartigen austauschbaren Phosphates, aber doch bedeutend weniger als bei dem Extrahieren ohne Ionenaustauscher, wobei das ausgelöste Phosphat ein weiteres Austauschen hemmen würde. Wenn man einen möglichst grossen Teil 
des austauschbaren Phosphates aus der Bodensubstanz zu extrahieren wünscht, muss man entweder eine möglichst stark austauschende Extraktionslösung oder im Verhältnis zu der Bodenprobe grosse Mengen Harz oder Extraktionslösung gebrauchen. Extreme sind jedoch in dieser Hinsicht zu vermeiden.

Eine zweckmässig verdünnte Kaliumkarbonatlösung ist offenbar besser dazu geeignet. Ausserdem verhindert das Karbonat die sekundäre Ausfällung der Phosphationen als Kalziumphosphat durch Ausfällen des in die Lösung ausgetauschten Kalziums.

Die aus der Bodensubstanz ausgetauschte Phosphatmenge ist eigentlich in Anwesenheit von Harz, wenigstens bei den von uns gebrauchten Mengen Harz und Lösung, von der Konzentration der Kaliumkarbonatlösung nicht sehr abhängig. Bei Anwendung von stärker konzentrierten Karbonatlösungen geht event. auch etwas nichtaustauschbares Phosphat und wenigstens organische Substanz in die Lösung, was natürlich nicht wünschenswert ist. Also besteht keine Ursache, konzentrierte Karbonatlösungen dabei anzuwenden.

Dagegen wirkt die Konzentration der Karbonatlösungen auf die Verteilung des ausgelösten Phosphates zwischen der Lösung und dem Harz beträchtlich ein. Je verdünnter eine Karbonatlösung ist, desto kleiner ist der Teil des ausgelösten Phosphates, der in der Lösung verbleibt. So z.B. befinden sich bei Anwendung von $3 \mathrm{~g}$ Harz (Amberlite IRA-400) in Karbonatform und $50 \mathrm{ml}$ Lösung in 0.01normaler Kaliumkarbonatlösung $6.25 \%$ und in 0.08-normaler Lösung $34.8 \%$ der ganzen ausgetauschten Phosphatmenge.

Wenn das Phosphat in der Lösung innerhalb des $\mathrm{pH}$-Bereiches 9-11 so gut wie ausschliesslich in Form zweiwertiger $\mathrm{HPO}_{4}$-Ionen vorkommt, herrscht zwischen den Aktivitäten der Phosphat- und Karbonationen in der Lösung und im Harz nach Donnan folgendes Gleichgewicht:

$$
\begin{aligned}
\frac{\left(\mathrm{HPO}_{4}^{--}\right)_{\text {Lösung }}}{\left(\mathrm{CO}_{3}^{--}\right)_{\text {Lösung }}}=\frac{\left(\mathrm{HPO}_{4}^{--}\right)_{\mathrm{Harz}}}{\left(\mathrm{CO}_{3}^{--}\right)_{\mathrm{Harz}}} \text { oder } \\
\left(\mathrm{HPO}_{4}^{-{ }^{--}}\right)_{\text {Lösung }}=\frac{\left(\mathrm{HPO}_{4}^{--}\right)_{\mathrm{Harz}} \cdot\left(\mathrm{CO}_{3}^{--}\right)_{\text {Lösung }}}{\left(\mathrm{CO}_{3}^{--}\right)_{\mathrm{Harz}}}
\end{aligned}
$$

Wenn nur ein kleiner Teil der Karbonationen des Harzes durch zweiwertige Phosphationen ersetzt wird, wie es sich bei der in Rede stehenden Bodenuntersuchung verhält ${ }^{1}$, kann man auch den Karbonatgehalt des Harzes als konstant betrachten, so dass die Aktivität der zweiwertigen Phosphationen in Lösung sowohl der Phosphataktivität im Harz als auch der Aktivität der Karbonationen in der Lösung direkt proportional ist. Nach meiner Untersuchung stimmt die oben-

1 Wenn die Austauschkapazität des Harzes Amberlite IRA-400, wie der Produzent angibt, 2.5 mg-Äqu pro $1 \mathrm{~g}$ ist, enthalten $3 \mathrm{~g}$ Harz in Karbonatform also $7.5 \mathrm{mg}$-Äqu Karbonat. Wenn aus einer Bodenprobe eine $10 \mathrm{mmol} \mathrm{H}_{3} \mathrm{PO}_{4}$ entsprechende Phosphatmenge (=710 $\left.\mathrm{mg} \mathrm{P}_{2} \mathrm{O}_{5}\right)$ pro $1 \mathrm{~kg}$ ausgelöst würde, die eine ziemlich grosse Menge ist, würde das bedeuten, dass bei Anwendung von $1 \mathrm{~g}$ Einwaage nur $0.02 \mathrm{mg}$ - $\mathrm{A} q u \mathrm{HPO}_{4}$-Ionen aus der Bodensubstanz getauscht wird, welche Menge nur etwa $0.27 \%$ von dem Totalkarbonatgehalt in $3 \mathrm{~g}$ Harz ausmacht. 
erwähnte Gleichung bei Anwendung des stark alkalischen Anionenaustauschers Amberlite IRA-400 in verdünnten Lösungen ebenso so gut überein, auch wenn anstatt der Aktivitäten der Phosphat- und Karbonationen deren Totalgehalte in Harz bzw. Lösung gebraucht werden.

Wenn die Extraktionslösung alkalisch, z.B. von einem pH-Wert von etwa 10, ist, wird ein starkalkalischer Anionenaustauscher dabei angewendet, weil die schwachalkalichen Harze sich in einem alkalischen Milieu zersetzen. Wenn das Harz in Karbonatform gebraucht und das festgelegte Phosphat auch mit einer stärker konzentrierten Karbonatlösung eluiert wird, ist das Harz nach einer kleinen Spülung mit der in Frage kommenden Extraktionslösung für eine neue Extraktion fertig.

Auch zwischen Bodensubstanz und Lösung besteht die obenerwähnte Regelmässigkeit nach Donnan. Aber inwieweit wir die Phosphat- und Karbonatkonzentrationen der verdünnten Gleichgewichtslösungen und die in Bodensubstanz gegen das ausgetauschte Phosphat — eingetauschte äquivalente Karbonatmenge für die Berechnung der ganzen austauschbaren Phosphatmenge im Boden auch in diesem Falle anwenden können, bedarf noch einer genauerer Untersuchung. Es ist klar, dass die berechnete Totalmenge des austauschbaren Phosphates um so genauer ist, je grösser die Phosphatmenge ist, die aus der Bodenprobe extrahiert wird.

In den obenbeschriebenen mittleren Teil des Glasrohres $D$ geht nur $3 \mathrm{~g}$ Harz. Nichts hindert daran, grössere Rohre zu verfertigen. Dann kan man das Verhältnis Harz: Bodenprobe vergrössern, ohne die Menge der Bodenprobe zu verklemern. Um den Fehler der Probenentnahme zu vermeiden, dürfen die Einwaagen nicht kleiner als $1 \mathrm{~g}$ sein. Je grösser die gebrauchte Harzmenge ist, desto grösser ist die Phosphatmenge, die auf einmal aus der Bodenprobe extrahiert werden kann.

Theoretisch müsste es möglich sein das austauschbare Phosphat aus der Bodensubstanz noch vollständiger aufgelöst zu bekommen, wenn man dazu eine verdünnte (z.B. 0.01-normale) Kaliumkarbonatlösung, deren pH-Wert durch Kaliumhydroxyd ein wenig gesteigert ist, gebraucht, da Hydroxylionen die Phosphationen verhältnismässig effektiver aus der Bodensubstanz als aus dem starkalkalischen Harz austauschen.

Dieses automatische Apparat kann möglicherweise auch zur Bestimmung von austauschbaren Kationen gebraucht werden.

\section{Zus a mmenfassung}

Es wird der Aufbau eines automatischen Apparates zur Bestimmung der austauschbaren Ionen im Boden durch Ionenaustauscher nebst der dabei angewandten Arbeitsmethodik und einigen Grundsätzen analytisch-technischer Art beschrieben.

Bei der Bestimmung des austauschbaren Phosphates im Boden mit diesem Apparat wurden in diesem Falle verdünnte Kaliumkarbonatlösungen in Gegenwart eines stark alkalischen Anionenaustauscherharzes (Amberlite IRA-400) in Karbonatform benutzt. 
Die bei der Verteilung des Phosphates zwischen diesen Lösungen und dem Harz geltenden Gesetzmässigkeiten wurden untersucht. Durch Anwendung von Harz kann man bei dem Extrahieren des austauschbaren Phosphates aus dem Boden mit milderen Lösungsmitteln zurechtkommen, was als ein Vorteil anzusehen ist.

Die Frage, auf welche ein möglichst richtiger Totalgehalt des austauschbaren Phosphates im Boden bei Anwendung dieses Apparates mit Harz durch Berechnung aus Gleichgewichtskonzentrationen oder annähernd durch direkte Bestimmung erreicht werden kann, wird diskutiert.

Professor Dr. Martti Salonen und Doktor Armo Teräsvuori danke ich für das erwiesene freundliche Interesse an dieser Arbeit.

\title{
LITERATUR
}

(1) JAnsson, T. 1943. Mjölkningsmaskinen, dess konstruktion och användning.

(2) LeEs, H. 1947. A simple automatic percolator. J. Agric. Sci. 37: 27-28.

(3) Sheard, R. W. \& Caldwell, A. G. 1955. The use of anionexchange resins in phosphorus fertility studies. Can. J. Agr. Sci. 35: $36-41$.

\section{SELOSTUS:}

AUTOMAATTINEN LAITE MAAN VAIHTUVIEN IONIEN, VARSINKIN FOSFAATTI-IONIEN, MÄ̈RITTÄMISTÄ VARTEN IONIVAIHTAJAN AVULLA

\author{
EUGEN BARKOFF
}

Maatalouskoelaitos, maanviljelyskemian ja -fysiikan osasto, Tikkurila

Kirjoituksessa esitetään tekijän suunnittelema automaattinen laite maan vaihtumiskykyisten ionien määrittämistä varten ioninvaihtajahartsia käyttäen. Uuttoliuos kiertää laitteessa jatkuvasti sykkivän imun voimalla, joka aikaansaadaan vakuumipumpulla ja lypsykoneen tykyttimellä, vuoronperään maan ja hartsin läpi. Kiertäessään liuos samalla vaihtaa maassa olevat ionit ensiksi liuokseen ja sitten hartsiin kunnes tasapaino kolmen yllämainitun komponentin välillä on saavutettu.

Laitteen käyttökelpoisuutta on kokeiltu uuttamalla maan vaihtuvaa fosfaattia laimeilla karbonaattiliuoksilla ja käyttämällä vahvasti-emäksistä hartsia Amberlite IRA-400 karbonaattimuodossa, joka emäksisissä karbonaattiliuoksissa ei hajaannu niinkuin heikosti-emäksiset hartsit. Vaihtuva fosfaatti saadaan uutetuksi maasta laimeilla liuoksilla täydellisemmin hartsin läsnäollessa kuin ilman hartsia, koska suurin osa maasta liuenneesta fosfaatista pidättyy hartsiin eikä siis ole estämässä maan fosfaatti-ionien jatkuvaa liuokseen vaihtumista. Tämä ilmeni suoritetuissa tutkimuksissa, joilla pyrittiin selvittämään fosfaatti-ionien jakaantumista hartsin ja eri väkevien karbonaattiliuosten kesken. Lisäksi on käsitelty mahdollisuutta saada ko. laitetta käyttäen oikea arvo maan vaihtuvan fosfaatin kokonaismäärälle joko laskemalla fosfaatti- ja karbonaatti-ionien tasapainoväkevyyksistä tai — edes likimain — suoraan määrittämällä. 